\title{
The use of various species of fungi in biofiltration of air contaminated with odorous volatile organic compounds
}

\author{
Milena Gospodarek ${ }^{1, *}$, Piotr Rybarczyk ${ }^{1}$, Anna Brillowska-Dąbrowska $^{2}$, \\ and Jacek Gębicki ${ }^{1}$ \\ ${ }^{1}$ Department of Process Engineering and Chemical Technology, Chemical Faculty, \\ Gdańsk University of Technology, ul. G. Narutowicza 11/12, 80-233 Gdansk, Poland \\ ${ }^{2}$ Department of Molecular Biotechnology and Microbiology, Chemical Faculty, \\ Gdańsk University of Technology, ul. G. Narutowicza 11/12, 80-233 Gdańsk, Poland
}

\begin{abstract}
Air pollution with odorous compounds is a significant social and environmental problem. This paper presents biological deodorization methods. The attention is focused on the application of biotrickling filters for air deodorization. Principles of their operation are discussed, indicating the key role of the selection of microorganisms responsible for the degradation of odorous compounds. A literature overview of the used fungal species is presented and the advantages of using fungi in comparison with bacteria are indicated. The results of experimental studies on the n-butanol removal in biotrickling filter are presented.
\end{abstract}

\section{Introduction}

Air pollution with odorous compounds is becoming a growing problem, especially for residents of large agglomerations and areas adjacent to industrial plants [1]. In order to reduce the emission of odorous substances into the atmosphere, various deodorization techniques are used, including physical methods (combustion), physicochemical methods (absorption, adsorption) and biological methods. Relatively low operating costs, low waste generation and the ability to clean large volumes of gases, containing low concentrations of odorous compounds, make the use of biological methods particularly beneficial $[2,3]$.

The biofiltration process consists in passing a polluted stream of gas through a filter bed, inhabited by microorganisms of various species (bacteria, fungi) [4]. The impurities diffuse from the gas phase to the so-called biofilm, being formed on the surface of the elements of the packed bed. The compounds adsorbed on the surface or absorbed in the biofilm are biodegraded and the air leaves the biofilter cleansed and free of unpleasant odours. The biofiltration process can be carried out in two main types of apparatus, i.e. in a conventional biofilter or in a biofilter with a trickled bed. In a conventional biofilter, the contaminated gas is initially humidified in a separate chamber, and only then it is passed through the biofilter bed. The packing of such a biofilter is most often made of natural

\footnotetext{
* Corresponding author: milena.gospodarek@pg.edu.pl
} 
materials, e.g. bark, cones or peat. The construction of a biotrickling filter, on the other hand, enables the process to be carried out in one apparatus. The filter bed, made of natural or synthetic materials, is regularly trickled with a liquid enriched with minerals necessary for the growth of microorganisms. The spraying liquid may circulate in a closed system and is subjected to periodic regeneration. Biofilters with a trickled bed allow easier control and regulation of the process, compared to conventional biofilters [5, 6].

Microorganisms are basic elements of biological processes of air purification [3]. Considering the deodorization mechanism described above, the selection of microorganisms colonizing the biofilter bed is crucial for the effective degradation of specific groups of chemical compounds. Depending on the type of air pollution, biofilters can be inoculated with selected strains or mixed consortia of microorganisms [7].

In recent years, many efforts have been made to develop optimal methods of air deodorisation using microorganisms. Fungi and bacteria deserve the most attention. The undoubted advantage of fungi used in biofilters, compared to other organisms, is a much higher resistance to drying out and acidification $[8,9]$. This makes it possible to carry out the process using fungi in relatively dry conditions, and thus it is possible to significantly reduce the thickness of the liquid layer adjacent to the biofilm that inhabits the microorganisms. Jin et al. [10] proved that fungi can be successfully used to remove hydrophobic compounds during biofiltration. Fungal biofilms are highly resistant to acidic conditions with low humidity, and they are able to absorb hydrophobic compounds from the gas phase faster and more effectively than in the case of bacterial biofilms.

In the case of filamentous fungi, compared to other fungal species, high pressure losses are more common. This is directly related to the problems of clogging and channelling of biofilter packed beds. The solution to these problems may be the use of mites in the biofiltration process, in addition to fungal organisms. Application of higher organisms allow to prevent a rapid drop in pressure, and thus result in a significant reduction in energy requirements. It has been proven that the mites used in biofilters are relatively easy to maintain during the biofiltration process, and may be successfully used as a means to control fungal biomass [10].

The advantage of fungi, as compared to bacteria, is that they have an aerial mycelium that significantly increases the surface area of the biofilm in the gas phase, thanks to which volatile odour compounds may be more efficiently captured. The key role in the growth and development of filamentous fungi is played by hydrophobins. These proteins secreted by fungi are involved in the formation of surface structures, as well as the formation of hyphae and their attachment to various types of hydrophobic surfaces. The removal of hydrophobic compounds is a current problem in biofiltration processes [11]. Hydrophobins are able to fulfill these functions due to their production by fungi on hydrophobic-hydrophilic surfaces. Thanks to these properties, fungi in biofilters can be used with high efficiency to remove impurities directly from the gas phase, and thus it is possible to overcome the resistance of mass transfer of hydrophobic compounds to aqueous liquids [12].

Table 1. Studies on the RE, and fungal community of current biofiltration systems for removing VOCs and odor waste gases.

\begin{tabular}{|c|c|c|c|c|}
\hline Predominate strain & Contaminant & Packing material & RE [\%] & Reference \\
\hline Sporothrix variecibatus & Styrene & Ceramic monolith & 95 & {$[15]$} \\
\hline $\begin{array}{c}\text { Sporothrix variecibatus, } \\
\text { Sporothix sp. }\end{array}$ & Acetone & Perlite & 75.6 & {$[16]$} \\
\hline Ascomycota strain & Trichloroethane & Wood chips & 59.2 & {$[17]$} \\
\hline Fusarium solani & Hexane & Perlite & - & {$[18]$} \\
\hline Ophiostoma sp. & Alpha-pinene & Perlite & 89 & {$[19]$} \\
\hline Cladophialophora sp. & Toluene & Perlite & 99 & {$[20]$} \\
\hline Fusarium oxysporum & Nitric oxide & Porous silicate pellets & 93 & {$[21]$} \\
\hline
\end{tabular}


The hydrophobicity of the mycelium surface can increase consistently as the amount of hydrophobic substrates increases. This phenomenon can be explained by the higher uptake of hydrophobic gas substances by fungi used in biofilters. Thanks to this, fungi are able to adapt to hydrophobic conditions [14]. Table 1 presents different studies where a fungus, as a dominant organism, was used for pollutant degradation.

As object of the study, n-butanol was chosen. It is due to its presence in gases emitted from municipal wastewater treatment plants or landfills [22, 23]. Additionally, the removal of n-butanol in biotrickling filters is rarely studied [24].

\section{Aim of the work}

In the experimental part of this paper, the effectiveness of n-butanol removal from air in a biotrickling filter as well as a preliminary assessment of the microbiological composition of the applied filter packing were investigated. The n-butanol removal efficiency was evaluated for the first twenty days of a biofilter operation, starting from introducing the n-butanol-air mixture flow through an activated filter bed. In the fifth day of the process, samples of a packed bed material for microbiological tests were taken. Selection of a period of 20 days of biofilter performance evaluation aims at verifying whether it is a sufficient time to reach steady-state conditions i.e. if the start-up period for the system is achieved.

\section{Calculations}

The effectiveness of n-butanol removal from air was assessed on the basis of the removal efficiency (RE), calculated according to equation (1):

$$
\left.R E=\left(C_{\text {in }}-C_{\text {out }}\right) / C_{\text {in }}\right) \times 100 \%
$$

where $C_{\text {in }}$ and $C_{\text {out }}$ stand for concentrations of n-butanol in the inlet and outlet gas streams, respectively. The value of empty bed residence time (EBRT) was calculated from formula (2):

$$
E B R T=V_{G} / Q
$$

where $V_{G}$ is the volume of packing in the biofilter and $Q$ is the volumetric flow rate of a gas phase.

\section{Experimental set-up}

The research was carried out in a two-section biotrickling filter, shown schematically in Figure 1. The biofilter was made of organic glass and packed with a commercially available mixture of peat and perlite (COMPO SANA, Compo, Germany). Dimensions and process parameters of a biofilter are given in Table 2.

In order to reduce the pressure drop across the packing, in each of the biofilter sections was placed loosely a layer of $10 \times 2.4 \mathrm{~mm}$ Raschig ceramic rings. A layer of $6 \times 1.5 \mathrm{~mm}$ Raschig rings was also placed over the peat layer to ensure uniform distribution of the trickling liquid in the biofilter packed bed. Trickling liquid was sprayed over the filter packing for $1 \mathrm{~min}$ per each $60 \mathrm{~min}$.

A stream of n-butanol and air from the gas mixture generator (1) was introduced via a pipe (2), equipped with an inlet gas sampling port (3), to the base of the biofilter (4). The purified gas leaves the biofilter with a pipe (5), equipped with an outlet gas sampling port 
(3). In countercurrent to the gas phase, at the top of the biofilter, a trickling liquid (7) was fed in via the pipe (6) taken from the trickling liquid reservoir (8) via a peristaltic pump (7). The trickling liquid circulates in a closed system, flowing from the biofilter through the pipe (9) to the reservoir (8). The trickling liquid was replaced by means of valves (10) and (11). The measurement of the gas pressure drop in the biofilter was made using a differential manometer (12).

Table 2. Basic dimensions and process parameters of a biotrickling filter.

\begin{tabular}{|c|c|c|}
\hline Parameter & Unit & Value \\
\hline Internal diameter of a biofilter & $\mathrm{dm}$ & 0.8 \\
\hline Total height of a two-section biofilter & $\mathrm{dm}$ & 6.8 \\
\hline Total volume of biofilter packing & $\mathrm{dm}^{3}$ & 2.5 \\
\hline Volumetric flow rate of gas phase & $\mathrm{dm}^{3} \mathrm{~min}^{-1}$ & 2.5 \\
\hline EBRT & $\mathrm{min}$ & 1 \\
\hline C $_{\text {in }}$ & $\mathrm{ppmv}$ & 200 \\
\hline Height of a Rasching rings layer: & $\mathrm{dm}$ & 0.3 \\
$10 \times 2.4 \mathrm{~mm}$ & & 0.2 \\
$6 \times 1.5 \mathrm{~mm}$ & $\mathrm{dm}^{3} \mathrm{~min}^{-1}$ & 0.2 \\
\hline Volumetric flow rate of trickling liquid & $\mathrm{Pa}$ & $549.4-902.5$ \\
\hline Pressure drop across the biofilter packing & & \\
during the experiment & &
\end{tabular}

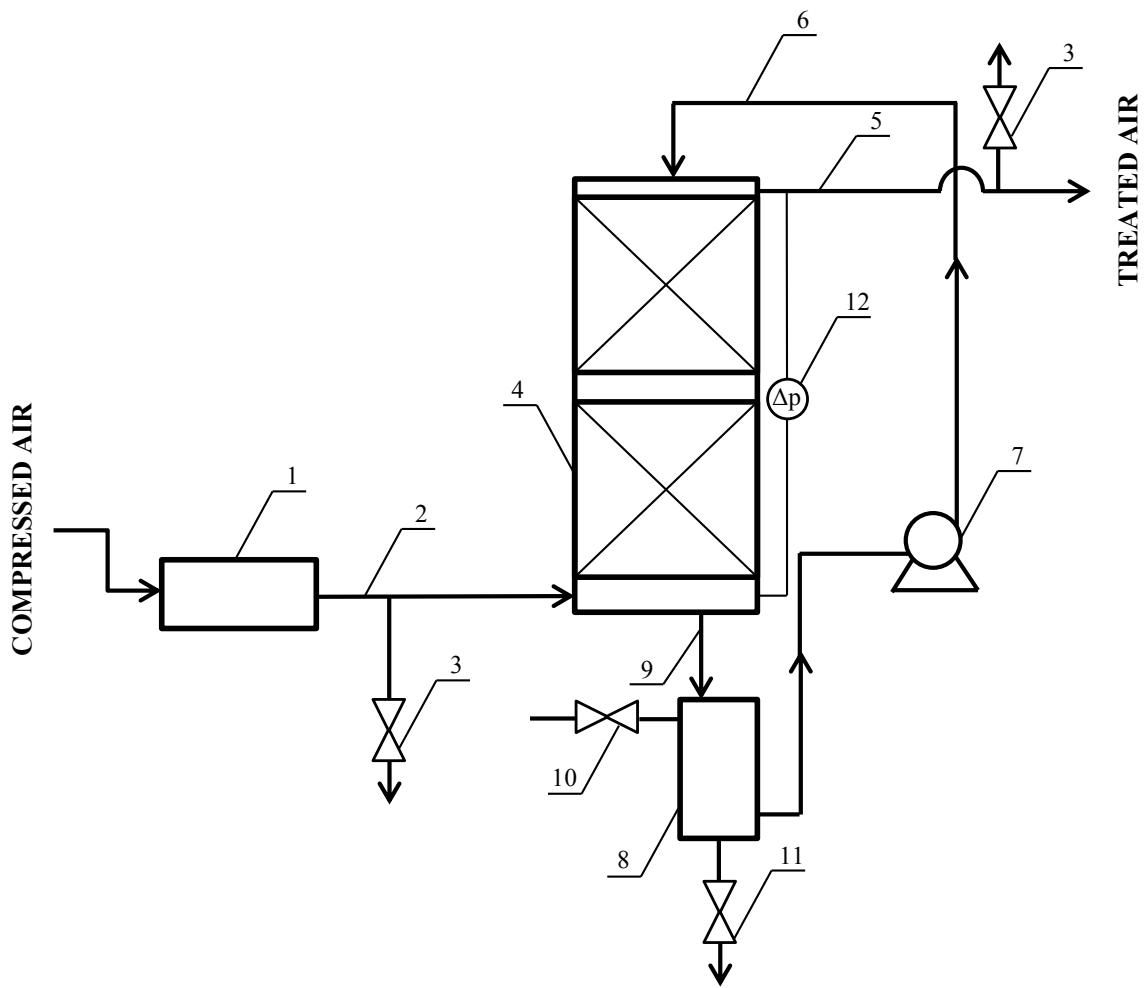

Fig. 1. Schematic diagram of the experimental set-up. 


\subsection{Biofiltration - process initiation and operation}

After placing a filter packing bed in the individual sections and having the bioreactor twisted, a flow of a clean air as well as a trickling of a liquid were introduced through a packed bed. The intensity of air and trickling liquid flows are as shown in Table 1. The activation of the biofilter packing lasted 4 days, and as a trickling liquid a Buffered Peptone Water medium was used. After activation of the bed, the flow of n-butanol and air mixture was initiated, further using a trickling liquid as described above. After 4 days of operation of the system, the liquid used was replaced with a fresh medium. The composition of the medium was changed and it is was as follows: $\mathrm{Na}_{2} \mathrm{HPO}_{4} \times 2 \mathrm{H}_{2} \mathrm{O}, \mathrm{KH}_{2} \mathrm{PO}_{4}, \mathrm{NaCl}$ and $\mathrm{NH}_{4} \mathrm{Cl}$. A trickling liquid of such a composition was changed every 4 days, up to day 20 of the process. During the tests, at least once a day, gas samples were taken from the inlet and outlet streams. The flow of the gas mixture stream at the inlet to the biofilter and the pressure drop on the filter bed were regularly controlled.

\subsection{Determination of $\mathbf{n}$-butanol concentration}

Concentrations of n-butanol in the gas samples at the inlet and outlet of the biofilter were determined using a VARIAN CP-3800 gas chromatograph with a flame ionization detector (FID). A $30 \mathrm{~m}$ long HP-5MS capillary column was used (inner diameter $0.25 \mathrm{~mm}$, film thickness $0.25 \mu \mathrm{m}$ ). Conditions of chromatographic analysis were as follows: carrier gas: nitrogen; carrier gas flow: $2 \mathrm{ml} / \mathrm{min}$; split: 3 ; oven temperature: $150^{\circ} \mathrm{C}$; injector temperature: $150^{\circ} \mathrm{C}$; FID temperature: $200^{\circ} \mathrm{C}$; total time of a single analysis: $5 \mathrm{~min}$. The analyzes were performed in triplicate and the results are presented for mean values.

\subsection{Microbiological methods}

To isolate the DNA of fungal strains, cultivation was carried out on Sabouraud medium. The plates were incubated at room temperature for about 2 days. In order to isolate the strains DNA, a short method developed by Brillowska-Dąbrowska et al. was used [25].

To obtain PCR products, ITS1 and ITS4primers were applied [26].The electrophoretic separation was carried out for 30 minute in a $\%$ agarose gel placed in 1xTAE buffer and at $100 \mathrm{~V}$.

$10 \mu \mathrm{l}$ of the mixture obtained after the PCR reaction was applied to each well. In order to compare the size of the products obtained, the DNA M100-500 marker was used. Electrophoresis results were recorded using the ChemiDOC apparatus.

\section{Results and discussion}

\subsection{Performance of a biotrickling filter}

Data presenting the changes of n-butanol removal efficiency with time of biofiltration is presented in Figure 2. Immediately after introducing the n-butanol-air mixture to the biofilter, RE about $37 \%$ is achieved. When the biofiltration is continued, an increase of n-butanol removal efficiency is observed. This may be a result of the adaptation of a filter medium to the composition of treated gas stream. In the second day of the process, values of $\mathrm{RE}$ reach the level of about $85 \%$. Further continuation of the process results in the stabilization of RE values in the range between about $85-92 \%$. During this period, the biofilter packing is further accommodated to the treated gas and the microbes present in the 
peat structure consume n-butanol as a source of carbon. Obtained results show that after 20 days of biofiltration, removal efficiency of $n$-butanol exceeds $90 \%$. This may indicate that the start-up period, characterized with a stable values of RE and assumed steady-state conditions in the filter bed, is achieved and a system is ready for changes of process parameters, including the variable inlet loading or EBRT[27]. Such investigations are currently performed in our research team.

The obtained removal of n-butanol is quite high ( $\mathrm{RE}>90 \%)$ and it is in accordance with literature data [24]. Because n-butanol is a hydrophilic volatile organic compounds, its high removal efficiency when applying aqueous trickling liquid is not surprising. Further investigations of the applied biotrickling filter system i.e. for the system employing natural packing materials should concentrate on the removal of hydrophobic compounds like toluene or cyclohexane. As excellent performance of natural packings in conventional biofilters is well known [6], further evaluation of the durability of the applied packing to biotrickling filters seem to be reasonable.

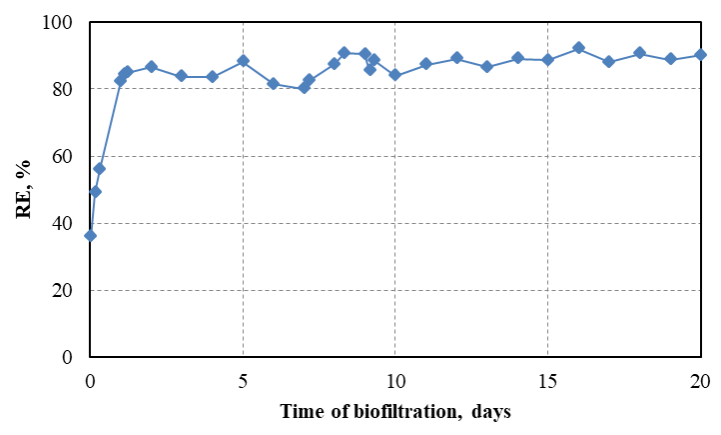

Fig. 2. Performance of a biotrickling filter packed with a mixture of peat and perlite.

\subsection{Evaluation of microbial composition of a biofilter packed bed}

Peat mixed with perlite was used as a biofilter packing material in this study. Peat is characterized by high wetland biodiversity. To date, 601 species of fungi from peatlands have been identified worldwide. The most popular are ascomycetes with 276 species (46\%), followed by basidiomycetes (40\%, 243 species), zygomycetes ( $9 \%, 55$ species) and chytridiomycetes $(4 \%, 26$ species). However, the most species-rich species are Penicillium (48 species), Galerina (41 species) and Mortierella (20 species). It should be noted that the 20 most common types of fungi constitute $42 \%$ of all fungi in peat bogs ( 252 from 601 species). Most fungi on peat bogs are saprobic organisms, so they participate in the decomposition of organic matter. Often this process is accompanied by a deodorization process[28].

For the taxonomic identification of fungi from the analyzed peat, cultivation was carried out on Sabouraud medium, which is a selective medium for the cultivation of fungi. Then a PCR reaction was carried out. A pair of primers with the sequences given in Table 3 were used for this purpose. The PCR reaction was carried out using the temperature-time profile described in Table 4.

Table 3. Primer sequences used in the PCR reaction.

\begin{tabular}{|c|c|}
\hline Name & Primer Sequence \\
\hline ITS1 & TCCGTAGGTGAACCTGCGG \\
\hline ITS4 & TCCTCCGCTTATTGATATGC \\
\hline
\end{tabular}


Table 4. Temperature-time profile of the PCR reaction.

\begin{tabular}{|c|c|c|c|}
\hline Response stage & Temperature $\left[{ }^{\circ} \mathbf{C}\right]$ & Time $[\mathbf{s}]$ & Number of cycles \\
\hline Initial denaturation & 95 & 180 & 1 \\
\hline Specific denaturation & 95 & 30 & \multirow{2}{*}{35} \\
\hline Attachment of starter & 57 & 30 & \\
\hline Elongation & 72 & 45 & 1 \\
\hline Final elongation & 72 & 300 & 1 \\
\hline Cooling & 4 & $\infty$ & \\
\hline
\end{tabular}

The Figure 3 presents the results of the electrophoretic separation of the amplified DNA fragments using the ITS1 and ITS4 primers for the isolates obtained. The authors of the manuscript anticipated obtaining single bands, which would indicate the isolation of DNA from a single microorganism. Unfortunately, in the case of three out of five tested samples (3-5), double bands where received, which indicates a failed attempt to obtain pure culture. Double bands indicate the presence of at least two species.
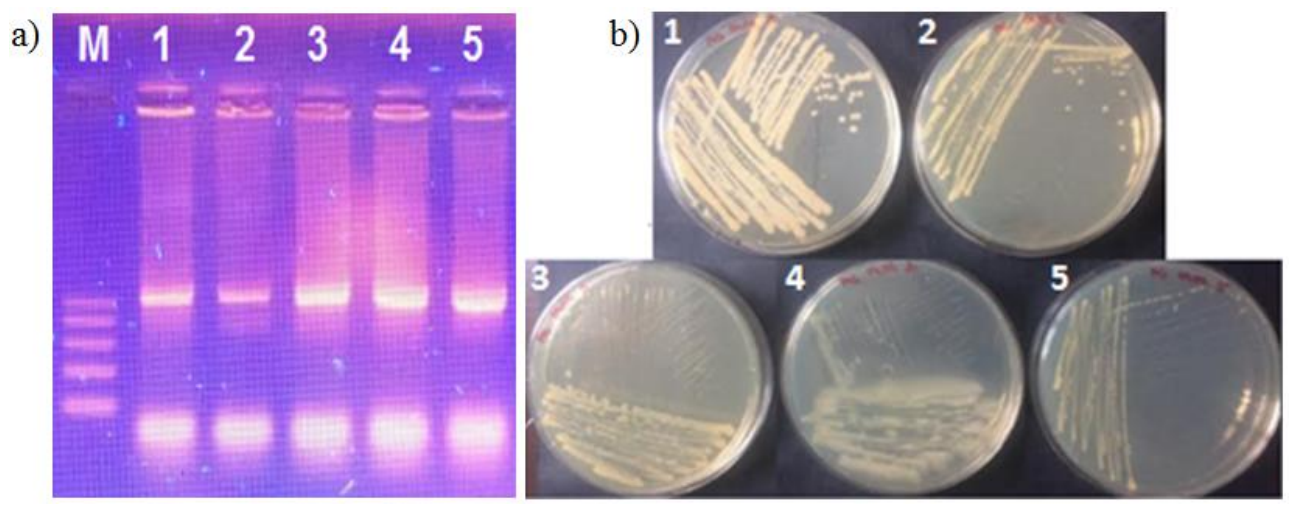

Fig. 3. (a) Result of electrophoretic separation of PCR reaction products for unidentified isolates. Agarose gel 2\%, separation time: 30 minutes, voltage 100V, M - marker size $100-500$ bp. (b) Fungi from the analyzed peat.

In the next step, a streak method was applied for the obtained cultures to confirm the presence of different species. The colonies found on plates 1 and 2 represent yeast, however cultures on plates 3, 4, 5 consist of yeast and molds (Figure 4). It confirmed the necessity of application of several streak plate cultures to obtain pure cultures.

\section{Conclusions}

Experimental results presented in this study indicate that n-butanol vapors may be effectively eliminated from air in a biotrickling filter packed with a mixture of peat and perlite. Removal efficiency of n-butanol exceeding $90 \%$ has been achieved after a period of 20 days of biofiltration. Such a period may be taken as a start-up period, indicating the stable conditions in the biofilter bed. Based on the literature review and the results of microbiological investigations it can be concluded that peat is inhabited by many species of microorganisms, including fungi. Further studies on the efficiency of removal of hydrophobic volatile organic compounds as well as the microbial compositions of a peat-packed biotrickling filter are suggested.

This work was financially supported by the project "INTERPHD2" No. POWR.03.02.00-IP.08-00DOC/16. 


\section{References}

1. P. Lewkowska, B. Cieślik, T. Dymerski, P. Konieczka, J. Namieśnik, Environ. Res. 151 (2016)

2. Q. Zhou, L. Zhang, J. Chen, B. Xu, G. Chu, J. Chen, Chem. Eng. J. 284 (2016)

3. K. Barbusinski, K. Kalemba, D. Kasperczyk, K. Urbaniec, V. J. Kozik, Clean. Prod. 152 (2017)

4. D. McNevin, J. Barford, Biochem. Eng. J. 5 (2000)

5. R. Lebrero, L. Hernández, R. Pérez, J. M. Estrada, R. J. Muñoz, Environ. Manage. 151 (2015)

6. P. Rybarczyk, B. Szulczyński, J. Gębicki, J. Hupka, Biochem. Eng. J. 141 (2019)

7. S. Mudliar, B. Giri, K. Padoley, D. Satpute, R. Dixit, P. Bhatt, R. Pandey, A. Juwarkar, A. J. Vaidya, Environ. Manage. 91 (2010)

8. H. Cox, Rijksuniversiteit te Groningen (1995)

9. J. W. van Groenestijn,W. N. van Heininge, N. J. Kraakm, Water Sci. Technol. 44 (2001)

10. J. R. Woertz, K. A. Kinney, N. J. R. Kraakman, W. N. M. van Heiningen, M. H. A. Eekert, Exp Appl Acarol. 27 (2003)

11. Y. Cheng, H. He, C. Yang, G. Zeng, X. Li, H. Chen, G. Yu, Biotechnol. Adv. 34 (2016)

12. H. A. B. Wösten, M.-A. van Wetter, L. G. Lugones, H. C. van der Mei, H. J. Busscher, J. G. H. Wessels, Curr. Biol. 9 (1999)

13. H. A. B. Wösten, Annu. Rev. Microbiol. 55 (2001)

14. A. Vergara-Fernández, B. van Haaren, S. Revah, Biotechnol. Lett. 28 (2006)

15. E. R. Rene, M. E. López, M. C. Veiga, C. Kennes, Bioresour. Technol. 101 (2010)

16. E. R. Rene, R. Spačková, M. C. Veiga, C. J. Kennes, Hazard. Mater. 184 (2010)

17. Y. Quan, H. Wu, C. Guo, Y. Han, C. Yin, Bioresour. Technol. 259 (2018)

18. S. Arriaga, S. Revah, Can. J. Civ. Eng. 36 (2009)

19. Y. Jin, L. Guo, M. C. Veiga, C. Kennes, Biotechnol. Bioeng. 96 (2007)

20. W. Woertz, M. van Heiningen, J. van Ee, W. van Heiningen, M. van Eekert, N. Kraakman, K. Kinney, J. van Groenestijn, Appl. Microbiol. Biotechnol. 58 (2002)

21. J. Woertz, K. Kinney, P. J. Szaniszlo, Air Waste Manage. Assoc. 51 (2001)

22. B. Szulczyński, P. Rybarczyk, J.Gębicki, Monatshefte für Chemie - Chem. Mon. 149 (2018)

23. B. Szulczyński, J. Gębicki, J. Namieśnik, Chemical Papers, 72 (2018)

24. T. Schmidt, W. Anderson, Environments, 4 (2017)

25. A. Brillowska-Dąbrowska, A. Świerkowska, D. M. Lindhardt Saunte, M. C. Med. Mycol. 48 (2010)

26. T. White, T. Bruns, S. Lee, Mycologia, 64 (1990)

27. H. Qian, Y. Cheng, C. Yang, S. Wu, G. Zeng, J. Xi, Environ. Sci. Pollut. Res. 25 (2018)

28. M. N. Thormann, A. V. Rice, Fungal Diversity, 24 (2007) 